\title{
Influence of Tinnitus-Control Sound Stimuli on Auditory Spectral and Temporal Resolution and Speech Perception in Hearing Aid Users
}

\author{
Hyun Joon Shim, MD, PhD, Yong Tae Cho, MD, Dong Hyun Kim, BS and Jung Ho Choi, MD \\ Department of Otorhinolaryngology-Head and Neck Surgery, Nowon Eulji Medical Center, Eulji University, Seoul, \\ Korea
}

\begin{abstract}
- ABSTRACT -
Background and Objectives: Hearing aids (HAs) with built-in sound generators can alleviate the discomfort of tinnitus in individuals with tinnitus and impaired hearing. However, it is unknown how tinnitus-control sound (TCS) affects speech perception in HA users. Therefore, this study aimed to evaluate the effect of HA TCS on auditory spectral resolution, temporal resolution, and speech perception in individuals with tinnitus and impaired hearing. Materials and Methods: The subjects were 13 elderly listeners (62.38 \pm 8.44 years, seven males, six females) with tinnitus and impaired hearing who had been using a receiver-in-canal HA with a built-in sound generator in everyday life for more than 3 months. The following psychoacoustic measurements were performed: spectral-ripple discrimination (SRD), temporal modulation detection (TMD), and speech recognition threshold in noise. The results obtained with HA-only and HA plus TCS conditions were compared. Results: There were no significant differences in SRD and TMD between the HA-only and HA plus TCS conditions ( $>0.05)$. However, the HA plus TCS conditions were associated with worse speech recognition than the HA-only conditions $(\mathrm{p}=0.027)$. Conclusions: TCS constrains speech perception in noise in individuals with tinnitus and impaired hearing, implying that the TCS introduces additional noise to existing tinnitus. Therefore, we recommend that individuals with tinnitus who use a HA with a built-in sound generator should turn off the sound generator in daily life, especially in noisy environments. (J Clinical Otolaryngol 2021;32:202-211)
\end{abstract}

KEY WORDS: Hearing aids; Sound generator; Speech perception; Spectral resolution; Temporal resolution.

\section{Introduction}

Sound therapy is one of the oldest modalities for treating tinnitus and is still widely used. Listening to an external sound masks the perception of tinnitus, and helps the individual to feel more comfortable than in a quiet environment. Various sounds, such as pure tones, narrow band noise, white noise, and notched music, have been used to shield tinnitus. ${ }^{1-5)}$ Jastreboff investigated habituation of tinnitus perception by supplying an external sound at the mixing point, where the tinnitus is mixed with the shielding sound instead of completely masking the tinnitus. ${ }^{6}$ Acoustic stimulation in individuals with tinnitus can decrease the discomfort induced by tinnitus, evoke positive feelings, and induce relaxation to facilitate long-term habituation to tinnitus. ${ }^{7}$ Moreover, specially designed sounds may restore the maladapted plastic change of the auditory cortex by altering the lateral inhibition or neural synchronization. ${ }^{5}$

The amplification of the external sound by a hearing aid (HA) not only masks the tinnitus itself, but also compensates for the hearing loss of individuals with tin-

Received: November 1, 2021 / Revised: November 23, 2021 / Accepted: December 3, 2021

Corresponding author: Hyun Joon Shim, Department of Otolaryngology, Eulji University School of Medicine, Nowon Eulji Medical Center, 68 Hangeulbiseok-Ro, Nowon-Gu, Seoul 01830, Korea

Tel: +82-2-970-8276 · Fax: +82-2-970-8275 · E-mail: eardoc11@naver.com 
nitus. ${ }^{8)}$ This may facilitate restoration of the maladapted plastic change of the auditory cortex by resupplying an auditory input. Sometimes, however, the sound amplified by the HA does not mask the tinnitus fully. In some individuals, the sound is insufficient to mask the tinnitus in quiet environments, and they may not want to wear their HA in quiet conditions. Therefore, many HA manufacturers have developed combination device comprising a HA and a built-in sound generator, which produces a tinnitus-control sound (TCS). The manufacturers often provide a program to change the device from a HA-only option to a HA plus TCS option. Several studies have demonstrated better therapeutic effects on tinnitus by using the combination device (i.e., HA plus sound generator) compared with a HA alone. ${ }^{4,9)}$ However, one concern about these combination devices is that the TCS may hinder the user's speech perception; however, it is unknown whether this is the case. Several studies have revealed that tinnitus functions as a central masker that constrains the individual's speech perception ability. ${ }^{10-13)}$ Therefore, TCSs might improve speech perception by unmasking the masking effect of tinnitus on speech perception. Auditory spectral resolution and temporal resolution are fundamental aspects of speech perception. Therefore, in this work, we evaluate the effect of TCS produced by HAs with built-in sound generators on auditory spectral resolution, temporal resolution, and speech- perception ability in individuals with tinnitus and impaired hearing.

\section{Materials and Methods}

\section{Subjects}

The study included 13 elderly subjects $(62.38 \pm 8.44$ years, seven males and six females) who had tinnitus and impaired hearing, and had been using HAs with built-in sound generators (10 unilateral HAs and three bilateral HAs) in everyday life for more than 3 months. They also used sound generators while using HAs. All of the subjects wore receiver-in-canal HAs (Unitron, Waterloo, ON, Canada). The TCS was made of white noise $(170-8,500 \mathrm{~Hz})$ and was set to match the mixing point similar to the loudness of tinnitus. Table 1 presents the demographics of subjects, the side and usage time of the HA, and the characteristics of tinnitus at the first visit. The mean pure tone average (PTA) of the right ears decreased from $58.44 \pm 8.56$ decibels hearing loss (dB HL) without the HA to $42.81 \pm 9.92 \mathrm{~dB}$ HL with the HA, and the mean PTA of the left ears decreased from $52.29 \pm 15.52 \mathrm{~dB}$ HL to $37.08 \pm 11.42 \mathrm{~dB}$ HL, respectively (Fig. 1). This study was approved by the Institutional Review Board of Nowon Eulji Medical Center, Seoul, Korea, and all of the subjects provided written informed consent.

\section{Procedure}

After using HAs with built-in sound generators for more than 3 months, three psychoacoustic measurements were performed to compare the results for the HA-only and HA plus TCS conditions: spectral-ripple discrimination (SRD), temporal modulation detection (TMD), and speech recognition threshold (SRT) in noise. The SRD test evaluated spectral resolution by measuring the subject's ability to discriminate a reversal in the phase of a ripple shape. TMD evaluated the subject's sensitivity to the temporal envelope by discriminating modulated noise from steady noise. The TMD test was conducted with modulation noises at 10 and $100 \mathrm{~Hz}$. To measure the SRT, equally difficult spondee words, spoken by a male speaker, were presented in the presence of speech-shaped, steady noise. The test are described in more detail in the Supplementary Procedures (Appendix 1). The stimuli were presented at 65 dBA. The stimuli were routed through an audiometer (Madsen Astera 2, GN Otometrics, Taastrup, Denmark) and presented by a loudspeaker placed $1 \mathrm{~m}$ in front of the subjects. All tests were conducted in a sound-attenuating booth (Acoustic systems, Austin, TX, USA). 
J Clinical Otolaryngol 2021;32:202-211

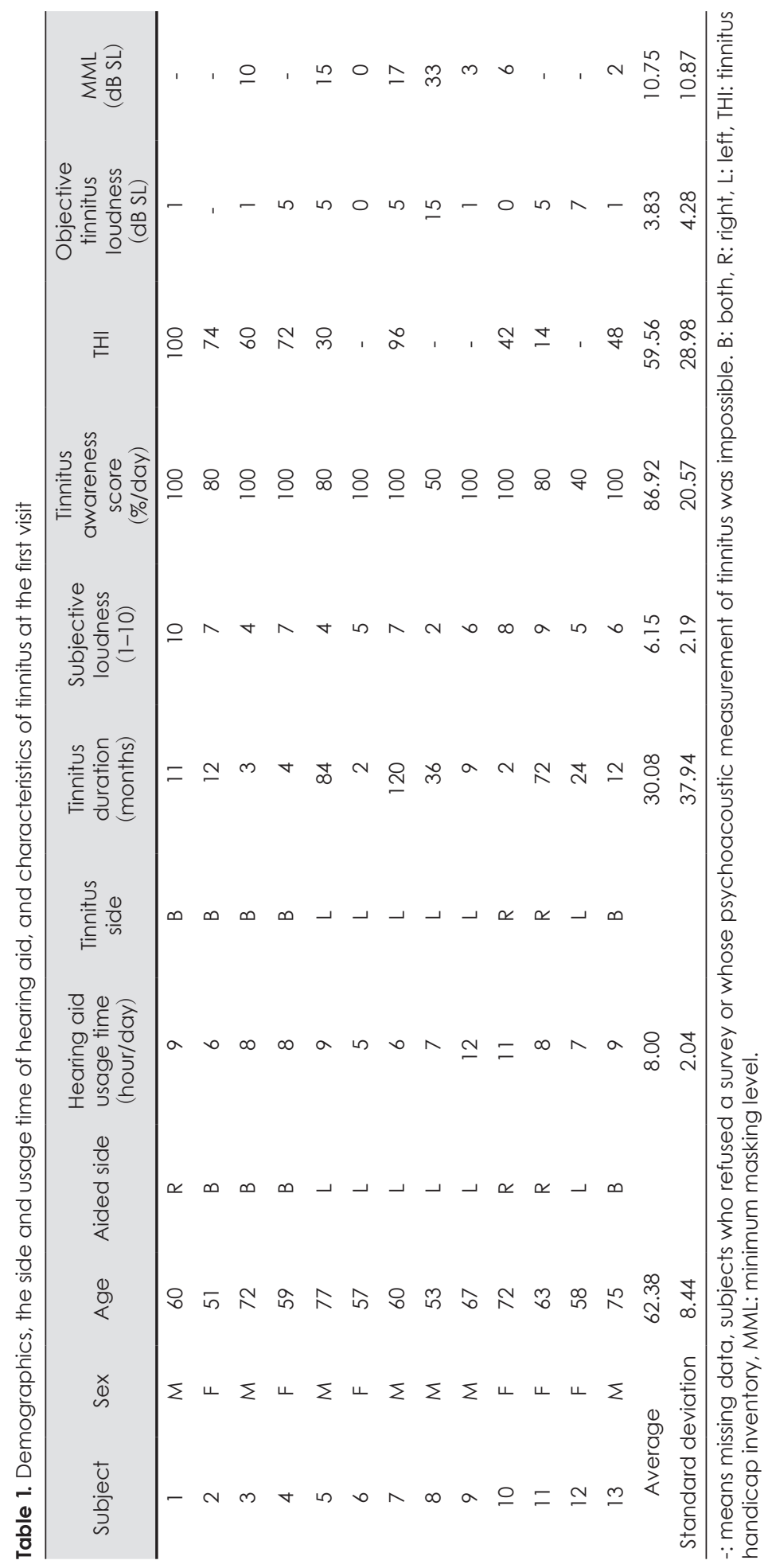



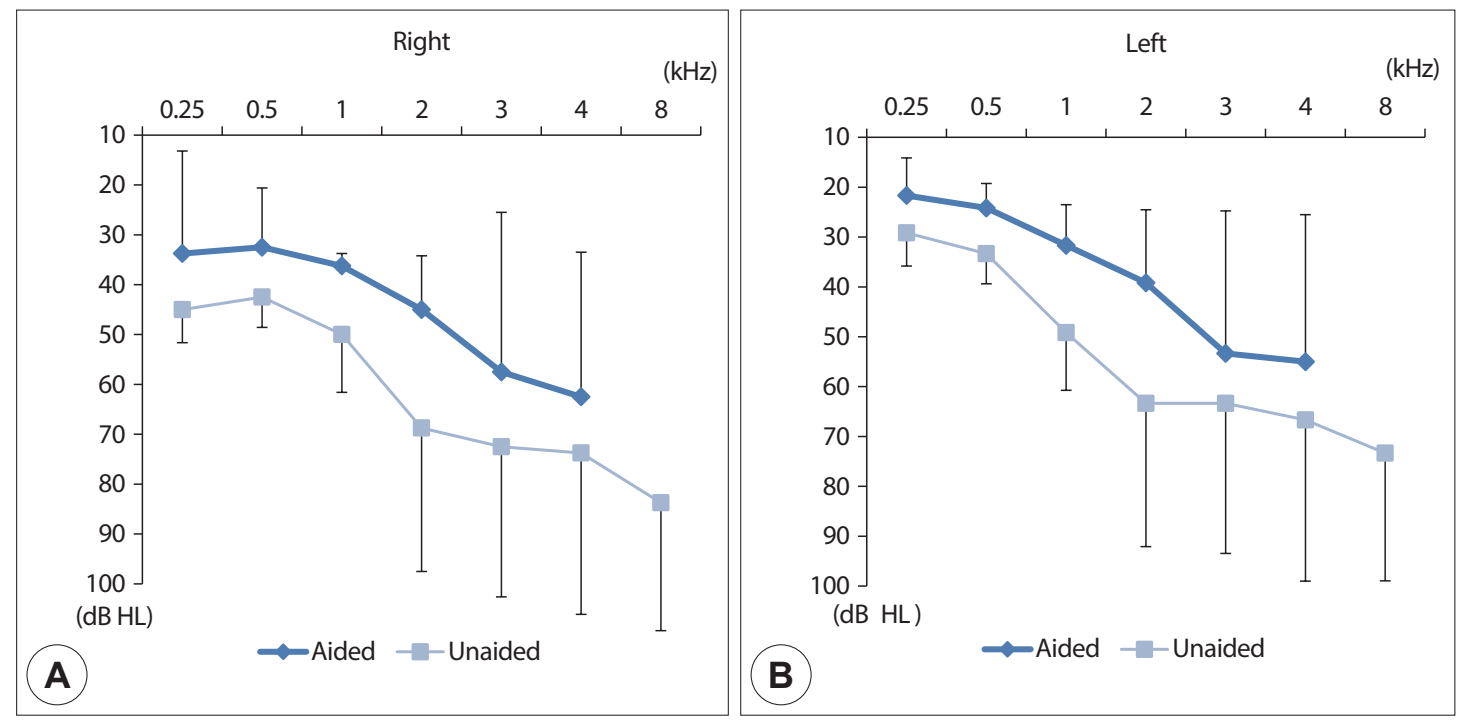

Fig. 1. Mean pure tone hearing thresholds of the 13 hearing aid users ( 10 unilateral and three bilateral hearing aids) in aided and unaided conditions. $\mathrm{dB} \mathrm{HL}$, decibels hearing loss.

\section{Analysis}

SPSS version 10 (SPSS, Chicago, IL, USA) was used for all statistical analyses. The results of the three tests were between the HA-only and HA plus TCS conditions using Wilcoxon's signed rank test, as appropriate. Correlations between the SRTs in noise and tinnitus characteristics were analyzed using Pearson's correlation coefficient.

\section{Results}

The comparisons of psychoacoustic test results for the HA-only and HA plus TCS conditions revealed no significant differences in the mean spectral ripple discrimination threshold $(1.41 \pm 0.78$ vs $1.50 \pm 0.78$, respectively, $\mathrm{Z}=-0.178, \mathrm{p}=0.859$ ), TMD threshold at $10 \mathrm{~Hz}$ $(-23.05 \pm 2.96$ vs $-23.79 \pm 4.79$, respectively, $Z=0.623$, $\mathrm{p}=0.533)$ and $100 \mathrm{~Hz}(-10.05 \pm 4.97 \mathrm{vs}-9.65 \pm 5.17$, respectively, $Z=-0.667 \mathrm{p}=0.505)$. However, the $S R T$ in noise (i.e., poor speech perception) was significantly greater in the HA plus TCS conditions than in the HA-only conditions $(2.70 \pm 6.49$ vs $1.45 \pm 5.94$, respec- tively, $Z=-2.194, p=0.028$, Fig. 2). Fig. 3 shows comparison of individual data for the three psychoacoustic tests. We also analyzed the correlations between SRTs in the HA plus TCS and HA-only conditions with various tinnitus characteristics, including subjective loudness, awareness time, duration, objective loudness, Tinnitus Handicap Inventory, and minimum masking level. However, the SRTs in both conditions were not significantly correlated with the tinnitus characteristics ( $>0.05)$.

\section{Discussion}

This study has revealed that TCS from a built-in sound generator constrains the HA user's speech perception ability in noise, implying that the TCS introduces additional noise to the existing tinnitus. The majority of studies of speech perception and tinnitus have demonstrated that tinnitus hinders speech perception, especially in noisy conditions, ${ }^{10-13)}$ except for one recent study by Zeng et al., ${ }^{14)}$ who suggested that tinnitus does not impair the perception of external sounds. In a pre- 


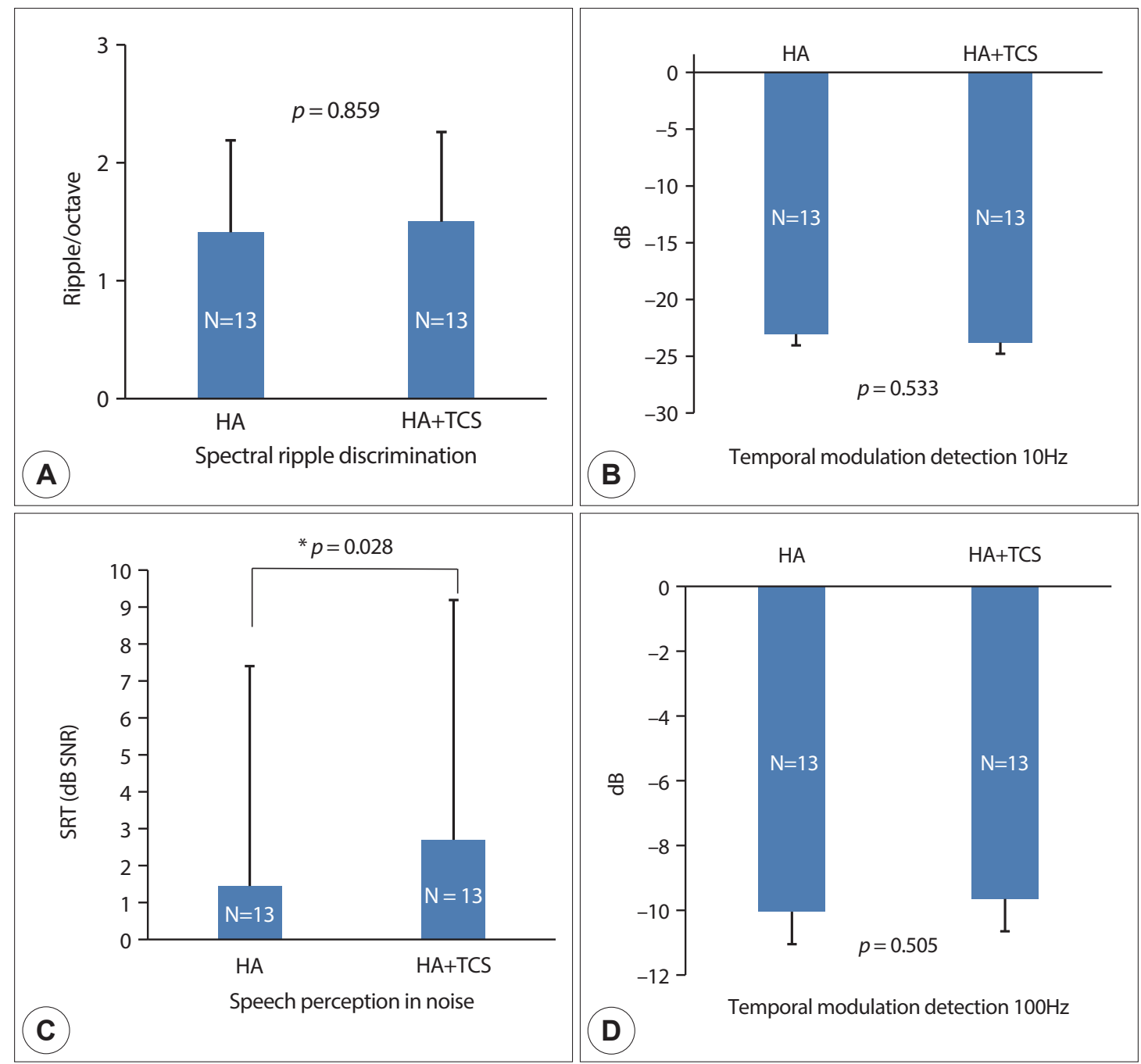

Fig. 2. Comparison of mean data from the psychoacoustic tests (spectral-ripple discrimination, temporal modulation detection at 10 and $100 \mathrm{~Hz}$, and speech perception in noise) between the hearing aid-only and hearing aid plus tinnitus-control sound conditions. HA: hearing aid, TCS: tinnitus-control sound, SRT: speech recognition threshold, SNR: signal-to-noise ratio.

vious study performed by the author's group, tinnitus constrained the subject's speech perception in noise, without affecting the spectral or temporal resolution of hearing. ${ }^{11)}$ The results imply that tinnitus may affect the central auditory system rather than the peripheral auditory system and may function as a central masker to degrade speech perception when individuals are listening to speech in the presence of background noise. Thus, it is hypothesized that if the TCS offsets the tinnitus, speech perception may improve under TCS conditions in individuals with tinnitus. However, the results of this study do not support this hypothesis. Rather, although TCS alleviates the discomfort of tinnitus, the loss of speech perception ability, as a function unique to HAs, is inevitable. Therefore, we recommend that individuals with tinnitus who use a HA with a built-in sound gen- 

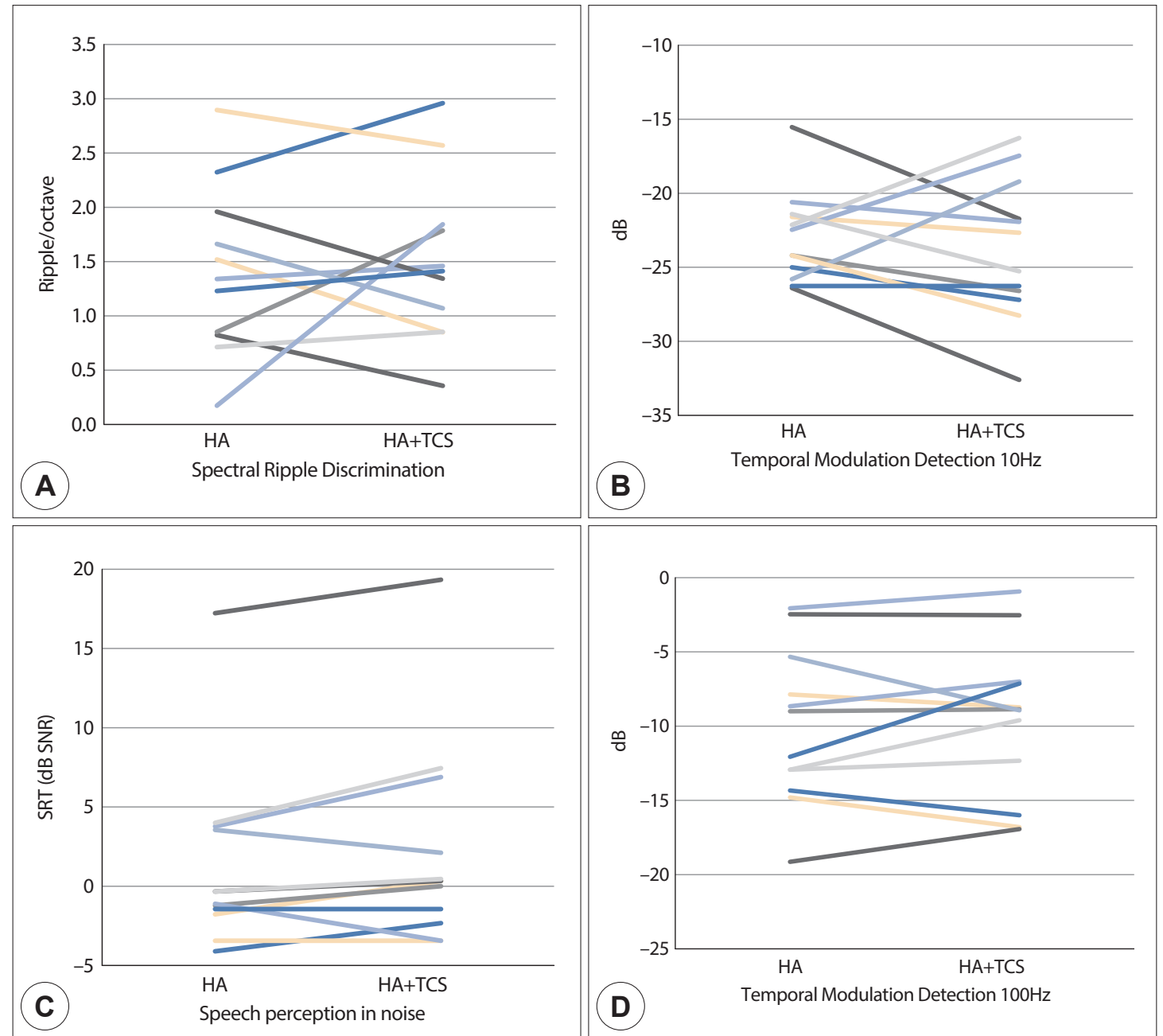

Fig. 3. Comparison of individual data for the three psychoacoustic tests (spectral-ripple discrimination, temporal modulation detection at 10 and $100 \mathrm{~Hz}$, and speech perception in noise) between the hearing aid-only and the hearing aid plus tinnitus-control sound conditions. HA: hearing aid, TCS: tinnitus-control sound, SRT: speech recognition threshold, SNR: signal-to-noise ratio.

erator should turn off the sound generator in daily life, especially in a noisy environment.

In this study, the TCS did not affect auditory spectral resolution or temporal resolution. Auditory spectral resolution primarily depends on the active movement of outer hair cells ${ }^{15)}$ and auditory temporal resolution is thought to be more strongly associated with central auditory processing than with auditory filtering. ${ }^{16,17)}$ Because the TCS was set to a loudness similar to that of tinnitus (only $3.8 \pm 4.3 \mathrm{~dB}$ sensation level of the tinnitus loudness in this study), it seems likely that the loudness of the TCS was insufficient to affect the spectral and temporal resolutions significantly. In several studies that used the same psychoacoustic tests as this study, the SRT in noise tended to change more sensitively than the SRD and TMD ${ }^{11,18)}$ The auditory spectral and temporal resolution largely affected speech perception and there were strong correlations between the spectral or tempo- 
ral resolutions and speech perception. ${ }^{19,20)}$ However, in a study that evaluated the effects of HAs on the spectral and temporal resolutions, the HAs improved speech perception in noise without changing the auditory spectral and temporal resolutions. ${ }^{18)}$

Most receiver-in-canal HAs are now equipped with built-in sound generators, and the TCS help to control tinnitus in affected individuals, especially in quiet environments. Henry et al. compared the improvement in tinnitus between using HAs only and HAs plus built-in sound generators. After 3 months, the mean reduction in Tinnitus Functional Index showed a greater trend towards significance in the combination device users than in HA alone users. However, the reduction in the Hearing Handicap Inventory Score was not significantly different between the two groups. ${ }^{4)}$ This suggests that the hearing benefit was not compromised by the addition of the TCS, in contrast to our results. However, hearing performance was evaluated by questionnaires in the study by Henry et al. rather than objective psychoacoustic tests, as in the present study. In another study, the relative efficacy of receiver-in-canal HAs, the same HAs with sound generators, and extended-wear, deep fit HAs (Lyric; Phonak, Stäfa, Swiss) on tinnitus control was evaluated. A clinically significant improvement in reaction to tinnitus was seen in all device groups, but these improvements did not differ across group. ${ }^{21)}$ In a Cochrane review using three randomized controlled studies compared HAs with sound generator with HAs only in terms of the effect of reducing tinnitus, no difference was found (standardized mean difference -0.15 , $95 \%$ confidence interval -0.52 to 0.22 ; 114 participants). ${ }^{22)}$ However, research on the comparison of HAs with sound generator and HAs has been conducted very rarely, making it difficult to conclude yet.

There were several limitations of this study. First, the sample size was small due to the difficulty in recruiting subjects. Second, data for unilateral HA and bilateral HA users were combined. Therefore, we cannot com- pletely rule out the possibility that some of the current results came out by chance, and further research using more sample size is needed in the future.

\section{Conclusion}

In individuals with tinnitus and hearing impairment, the TCS from the HAs constrained their speech perception ability in noise. This implies that the TCS introduces additional noise to the existing tinnitus, thus degrading the amplified speech signals generated by the HAs, even though the TCS can help alleviate the discomfort of tinnitus. Therefore, it is recommended that individuals with tinnitus who use a HA with a built-in sound generator should turn off the sound generator in daily life, especially in noisy environments.

\section{Acknowledgements}

The test program was created with the help of the Rubinstein Laboratory at the University of Washington (Seattle, WA, USA).

\section{Funding Information}

This work was supported by the National Research Foundation of Korea (NRF) grant funded by the Korean government (NRF2020R1I1A3071587).

\section{Conflicts of Interest}

No potential conflict of interest relevant to this article was reported.

\section{ORCID}

Hyun Joon Shim https://orcid.org/0000-0001-9719-6959

Yong Tae Cho https://orcid.org/0000-0001-6454-4670

Dong Hyun Kim https://orcid.org/0000-0003-0812-6751

Jung Ho Choi https://orcid.org/0000-0003-0722-2741

\section{Author Contribution}

Conceptualization: Shim HJ.

Data curation: Cho YT, Kim DH.

Formal analysis: Cho YT.

Funding acquisition: Shim HJ.

Methodology: Shim HJ, Kim DH.

Writing - original draft: Shim HJ, Cho YT.

Writing - review \& editing: Shim HJ, Cho YT, Kim DH, Choi $\mathrm{JH}$. 


\section{Ethics Approval}

This study was approved by the Institutional Review Board of Eulji Medical Center, Seoul, Korea (EMCS 2016-01-012), and all participants provided written informed consent.

\section{References}

1) Vernon J, Griest S, Press L. Attributes of tinnitus and the acceptance of masking. Am J Otolaryngol 1990;11(1):44-50.

2) Feldmann H. Homolateral and contralateral masking of tinnitus by noise-bands and by pure tones. Audiology 1971;10(3):138-44

3) Hobson J, Chisholm E, El Refaie A. Sound therapy (masking) in the management of tinnitus in adults. Cochrane Database Syst Rev 2012;11(11):CD006371.

4) Henry JA, Frederick M, Sell S, Griest S, Abrams H. Validation of a novel combination hearing aid and tinnitus therapy device. Ear Hear 2015;36(1):42-52.

5) Okamoto H, Stracke H, Stoll W, Pantev C. Listening to tailor-made notched music reduces tinnitus loudness and tinnitus-related auditory cortex activity. Proc Natl Acad Sci USA 2010;107(3):1207-10.

6) Jastreboff PJ, Hazell JWP. Tinnitus retraining therapy: implementing the neurophysiological model. Cambridge: Cambridge University Press; 2008.

7) Hann D, Searchfield GD, Sanders M, Wise K. Strategies for the selection of music in the short-term management of mild tinnitus. Aust N Z J Audiol 2008;30(2):129-40.

8) Vanneste S, van Dongen M, De Vree B, Hiseni S, van der Velden E, Strydis C, et al. Does enriched acoustic environment in humans abolish chronic tinnitus clinically and electrophysiologically? A double blind placebo controlled study. Hear Res 2013;296:141-8.

9) Jalilvand H, Pourbakht A, Haghani H. Hearing aid or tinnitus masker: which one is the best treatment for blast-induced tinnitus? The results of a long-term study on 974 patients. Audiol Neurotol 2015;20(3):195-201.

10) Ryu IS, Ahn JH, Lim HW, Joo KY, Chung JW. Evaluation of masking effects on speech perception in patients with unilateral chronic tinnitus using the hearing in noise test. Otol Neurotol 2012;33(9):1472-6.

11) Moon IJ, Won JH, Kang HW, Kim DH, An YH, Shim HJ. Influence of tinnitus on auditory spectral and temporal resolution and speech perception in tinnitus patients. J Neurosci 2015;35(42):14260-9.

12) Gilles A, Schlee W, Rabau S, Wouters K, Fransen E, Van de Heyning P. Decreased speech-in-noise understanding in young adults with tinnitus. Front Neurosci 2016;10:288.
13) Tai Y, Husain FT. Right-ear advantage for speech-innoise recognition in patients with nonlateralized tinnitus and normal hearing sensitivity. J Assoc Res Otolaryngol 2018;19(2):211-21.

14) Zeng FG. An active loudness model suggesting tinnitus as increased central noise and hyperacusis as increased nonlinear gain. Hear Res 2013;295:172-9.

15) Oxenham AJ, Bacon SP. Cochlear compression: perceptual measures and implications for normal and impaired hearing. Ear Hear 2003;24(5):352-66.

16) Eggermont JJ. Temporal modulation transfer functions in cat primary auditory cortex: separating stimulus effects from neural mechanisms. J Neurophysiol 2002;87(1):305-21.

17) Bao S, Chang EF, Woods J, Merzenich MM. Temporal plasticity in the primary auditory cortex induced by operant perceptual learning. Nat Neurosci 2004;7(9):974-81.

18) Kwak MY, Kang YK, Kim DH, An YH, Shim HJ. Further beneficial effect of hearing aids on speech recognition performance besides amplification: importance of the restoration of symmetric hearing. Otol Neurotol 2018;39(8):e618-26.

19) Baer T, Moore BC. Effects of spectral smearing on the intelligibility of sentences in noise. J Acoust Soc Am 1993;94(3):1229-41.

20) Henry BA, Turner CW, Behrens A. Spectral peak resolution and speech recognition in quiet: normal hearing, hearing impaired, and cochlear implant listeners. J Acoust Soc Am 2005;118(2):1111-21.

21) Henry JA, McMillan G, Dann S, Bennett K, Griest S, Theodoroff S, et al. Tinnitus management: randomized controlled trial comparing extended-wear hearing aids, conventional hearing aids, and combination instruments. J Am Acad Audiol 2017;28(6):546-61.

22) Sereda M, Xia J, El Refaie A, Hall DA, Hoare DJ. Sound therapy (using amplification devices and/or sound generators) for tinnitus. Cochrane Database Syst Rev 2018;12(12): CD013094.

23) Won JH, Drennan WR, Rubinstein JT. Spectral-ripple resolution correlates with speech reception in noise in cochlear implant users. J Assoc Res Otolaryngol 2007;8(3):384-92.

24) Byrne D, Dillon H, Tran K, Arlinger S, Wilbraham K, Cox $\mathrm{R}$, et al. An international comparison of long-term average speech spectra. J Acoust Soc Am 1994;96(4):2108-20.

25) Levitt H. Transformed up-down methods in psychoacoustics. J Acoust Soc Am 1971;49:467-77.

26) Won JH, Drennan WR, Nie K, Jameyson EM, Rubinstein JT. Acoustic temporal modulation detection and speech perception in cochlear implant listeners. J Acoust Soc Am 2011; 130(1):376-88. 


\section{Appendix 1. Supplementary Procedures}

\section{Spectral-ripple discrimination (SRD) test}

The SRD test was performed as described by Won et al. ${ }^{23)}$ To create spectral-ripple stimuli, the following equation was used:

$$
s(t)=\sum_{i=1}^{2555} 10^{D} \times\left\{a b s\left[\sin \left(\pi \times R \times F_{i}+\varnothing\right)\right]\right\} / 20 \times \sin \left(2 \times \pi \times 100 \times 50^{\frac{i-1}{2555}} \times t+\varphi_{i}\right),
$$

in which $\mathrm{D}$ is the ripple depth in $\mathrm{dB}, \mathrm{R}$ is ripples/octave, $\mathrm{F}_{\mathrm{i}}$ is the number of octaves at the ith component frequency (i.e., $\left.\left[i-1 \log _{10}(50)\right] /\left[200 \log _{10}(2)\right]\right), \phi$ is the spectral modulation starting phase in radians, $t$ is time in seconds, $\varphi_{1}$ is the randomized phase in radians (ranging between 0 to $2 \pi$ ) for each of the 2,555 pure-tone components. A ripple depth (D) of $30 \mathrm{~dB}$ was used. For the reference stimulus, the spectral modulation starting phase of the full-wave-rectified sinusoidal spectral envelope was set to zero radians, and for the "oddball" stimulus, the phase was set to $\pi / 2$ radians. The pure tones were spaced equally on a logarithmic frequency scale with a bandwidth of 100-4,991 Hz, ensuring a clear representation of the spectral peaks and valleys for stimuli with higher ripple densities. The ripple peaks were spaced equally on a logarithmic frequency scale. The stimuli had a total duration of $500 \mathrm{~ms}$ and were ramped with $150 \mathrm{~ms}$ linear rise/fall times. The stimuli were filtered with a long-term, speech-shaped filter that was created in CoolEdit 2000, with parameters specified in accordance with the findings of Byrne et al. ${ }^{24)}$ The order of presentation of the three tokens was randomized, and the participant's task was to select the "oddball" stimulus. No feedback was provided for this test. The stimuli were presented at $65 \mathrm{dBA}$. To measure SRD thresholds, a three-interval, three-alternative forced-choice (3-AFC) paradigm with an adaptive two-up and one-down procedure was used. The ripple density was varied between 0.125 and 11.314 ripples per octave in equal-ratio steps of 1.414 in an adaptive manner with 13 reversals that converges to the $70.7 \%$ correct point ${ }^{25}$. A level roving of $1-8 \mathrm{~dB}$ (in 1-dB increments) was randomly selected for each interval in the three-interval task. The SRD threshold for each adaptive run was calculated as the geometric mean of the last eight reversals of 13 reversals. The SRD threshold was determined by averaging the threshold from three testing runs.

\section{Temporal modulation detection (TMD) test}

The TMD test was performed using the method described by Won et al. ${ }^{26)}$ For the modulated stimuli, sinusoidal amplitude modulation was applied to a fresh wideband white noise carrier for each presentation. Two modulation frequencies were used: 10 and $100 \mathrm{~Hz}$. The stimuli were presented at $65 \mathrm{dBA}$. The stimulus duration for both modulated and unmodulated signals was 1 second. The modulated and unmodulated signals were gated on and off with 10-ms linear ramps and they were concatenated with no gap between the two signals. The TMD threshold was measured using a 1-interval, 2-AFC paradigm. One of the intervals contained modulated noise, and the other interval consisted of steady noise. Participants were asked to identify the interval which contained the modulated noise or "fluctuating" sound over time. A two-down, one-up adaptive procedure was used to measure TMD threshold. A visual feedback was provided following each presentation. The TMD thresholds (in $\mathrm{dB}$ ) relative to $100 \%$ modulation (i.e., $20 \log _{10}\left(\mathrm{~m}_{\mathrm{i}}\right)$ ) were obtained, where $\mathrm{m}_{\mathrm{i}}$ indicates the modulation index. The adaptive tracking procedure began with a modulation depth of $100 \%$ and changed in steps of $4 \mathrm{~dB}$ from the first to the fourth reversal, and $2 \mathrm{~dB}$ for the next 10 reversals. The TMD threshold for each adaptive test run was calculated as the mean of the final 10 reversals. 
The TMD threshold was determined by averaging the thresholds from three separate test runs.

\section{Speech recognition threshold in noise (SRT) test}

To measure SRTs, equally difficult spondee words, spoken by a male speaker, were presented in noise, which was spectrally shaped to have the same long-term power spectrum as the spondees. In all trials, the masker was gated on and off with 50-ms linear ramps $500 \mathrm{~ms}$ before and $50 \mathrm{~ms}$ after the target spondees. The mixture of the target spondee and masker stimuli was presented by a loudspeaker. SRTs corresponding to $50 \%$ intelligibility were measured using a one-up, one-down adaptive procedure. Each run started with a signal-to-noise ratio (SNR) of $6 \mathrm{~dB}$, for which participants were easily able to identify the spondee correctly. If a participant correctly repeated the spondee, the SNR for the next spondee was decreased; otherwise, the SNR was increased. No feedback was provided for this test. The stimuli were presented at $65 \mathrm{dBA}$. An initial step size of $4 \mathrm{~dB}$ was used for the first two reversals in the adaptive track, after which the step size was fixed at $2 \mathrm{~dB}$ for the next six reversals. When a participant showed a total 8 reversals, the adaptive run ended. The SRT for a given run was based on the average of the SNRs at each of the last six reversals of 8 reversals in the adaptive track. No spondee was repeated for any participant. Three adaptive runs were completed. The final SRT for each participant was taken as the mean of three separate adaptive runs. 